\title{
Big data managing in a landslide early warning system: experience from a ground-based interferometric radar application
}

\author{
Emanuele Intrieri ${ }^{1}$, Federica Bardi ${ }^{1}$, Riccardo Fanti ${ }^{1}$, Giovanni Gigli $^{1}$, Francesco Fidolini ${ }^{2}$, Nicola Casagli $^{1}$, \\ Sandra Costanzo ${ }^{3}$, Antonio Raffo ${ }^{3}$, Giuseppe Di Massa ${ }^{3}$, Giovanna Capparelli ${ }^{3}$, and Pasquale Versace ${ }^{3}$ \\ ${ }^{1}$ Department of Earth Sciences, University of Florence, via La Pira 4, 50121, Florence, Italy \\ ${ }^{2}$ Pizzi Terra srl, via di Ripoli 207H, 50126, Florence, Italy \\ ${ }^{3}$ Department of Informatics, Modeling, Electronics and System Engineering, University of Calabria, Ponte Pietro Bucci, \\ Cube 41b, 87036, Arcavacata di Rende (CS), Italy
}

Correspondence to: Emanuele Intrieri (emanuele.intrieri@unifi.it)

Received: 19 May 2017 - Discussion started: 31 May 2017

Revised: 25 August 2017 - Accepted: 29 August 2017 - Published: 6 October 2017

\begin{abstract}
A big challenge in terms or landslide risk mitigation is represented by increasing the resiliency of society exposed to the risk. Among the possible strategies with which to reach this goal, there is the implementation of early warning systems. This paper describes a procedure to improve early warning activities in areas affected by high landslide risk, such as those classified as critical infrastructures for their central role in society.

This research is part of the project "LEWIS (Landslides Early Warning Integrated System): An Integrated System for Landslide Monitoring, Early Warning and Risk Mitigation along Lifelines".

LEWIS is composed of a susceptibility assessment methodology providing information for single points and areal monitoring systems, a data transmission network and a data collecting and processing center (DCPC), where readings from all monitoring systems and mathematical models converge and which sets the basis for warning and intervention activities.

The aim of this paper is to show how logistic issues linked to advanced monitoring techniques, such as big data transfer and storing, can be dealt with compatibly with an early warning system. Therefore, we focus on the interaction between an areal monitoring tool (a ground-based interferometric radar) and the DCPC. By converting complex data into ASCII strings and through appropriate data cropping and average, and by implementing an algorithm for line-of-sight correction, we managed to reduce the data daily output without compromising the capability for performing.
\end{abstract}

\section{Introduction}

Urbanization, especially in mountain areas, can be considered a major cause for high landslide risk because of the increased exposure of elements at risk. Among the elements at risk, important communication routes, such as highways, can be classified as critical infrastructures (CIs), since their rupture can cause chain effects with catastrophic damages on society (Geertsema et al., 2009; Kadri et al., 2014). On the other hand, modern society is more and more dependent on CIs and their continuous efficiency (Lebaka et al., 2016), and this has increased their value over the years. The result is a higher social vulnerability in the face of loss of continuous operation (Kröger, 2008). The main objective was to improve the social preparedness for the growing landslide risk, according to the suggestions of several authors (Gene Corley et al., 1998; Baldridge and Marshall, 2011; Urlainis et al., 2014, 2015). This led to the development of several approaches and frameworks for increasing the resiliency of society exposed to the risk (Kröger, 2008; Cagno et al., 2011, and references therein). The resiliency policy involves not only prevention activities but also, and more importantly, those activities needed to maintain functionality after disruption (Snyder and Burns, 2009) and to promptly alert people of incoming catastrophes in order to protect them and prepare for a possible damaging of the endangered CI. Among these activities, the implementation of integrated landslides early warning systems (i.e., LEWIS: Landslides Early Warning Integrated System: An Integrated System for Landslide Monitoring, Early Warning and Risk Mitigation along Life- 
lines; Versace et al., 2012; Costanzo et al., 2016) reveals its increasing importance.

In this context, the methodology described in this paper has been conceived; it has been tested and validated on a portion of an Italian highway that is affected by landslides and that was selected as a case study: it is located in southern Italy, along a section of the A16 highway, an important communication route that connects Naples to Bari, where a ground-based interferometric synthetic aperture radar (GBInSAR) has been installed at the test site in order to obtain spatial monitoring data.

One of the main drawbacks of advanced instruments such as GB-InSAR is how to handle the large data flow deriving from continuous real-time monitoring. The issue is to reduce the capacity needed for analyzing, transmitting and storing big data without losing important information. The main feature of this paper is indeed the management of monitoring data in order to filter, correct, transfer and access them compatibly with the needs of an early warning system.

\section{Materials and methods}

\subsection{GB-InSAR}

The GB-InSAR is composed of a microwave transceiver mounted on a linear rail (Tarchi et al., 1997; Rudolf et al., 1999; Tarchi et al., 1999). The system used is based on a continuous-wave-stepped-frequency radar, which moves along the rail at millimeter steps in order to perform the synthetic aperture: the longer the rail, the higher the cross-range resolution. The microwave transmitter produces, step by step, continuous waves around a central frequency, which influences the cross-range resolution and determines the interferometric sensitivity, i.e., the minimum measurable displacement, usually largely smaller than the corresponding wavelength.

The radar produces complex radar images containing the information relative to both phase and amplitude of the microwave signal backscattered by the target (Bamler and Hartl, 1998; Antonello et al., 2004). The amplitude of a single image provides the radar reflectivity of the scenario at a given time, while the phase of a single image is not usable. The technique that enables to retrieve displacement information is called interferometry and requires the phase from two images. In this way, it is possible to elaborate a displacement map relative to the elapsed time between the two acquisitions.

The main added value of GB-InSAR is its capability to blend the boundary between mapping and monitoring, by computing 2-D displacement maps in near-real time. The use of this tool to monitor structures, landslides, volcanoes and sinkholes is widely documented (Calvari et al., 2016; Di Traglia, 2014; Intrieri et al., 2015; Bardi et al., 2016, 2017; Martino and Mazzanti, 2014; Severin et al., 2014; Tapete et al., 2013), as well as for early warning and forecasting (Intrieri et al., 2012; Carlà et al., 2016a, b; Lombardi et al., 2016).

GB-InSAR systems probably reveal their full potential in emergency conditions. They are transportable and only require from a few tens of minutes to a few hours to be installed (depending on the logistics of the site). Moreover, they can detect "near-real-time" area displacements, without accessing the unstable area, $24 \mathrm{~h}$ a day and in all weather conditions (Del Ventisette et al., 2011; Luzi, 2010; Monserrat et al., 2014). On the other hand, some limitations reduce the GBInSAR technique applicability: first of all, the scenario must present specific characteristics in order to reflect microwave radiations, maintaining high coherence values (Luzi, 2010; Monserrat et al., 2014); only a component of the real displacement vector can be identified (i.e., the component parallel to the sensor's line of sight); and maximum detectable velocities are connected to the time that the system needs to obtain two subsequent acquisitions. Sensors need power supply that, for long-term monitoring, cannot be replaced by batteries, generators or solar panels.

With the specific aim of performing the function of an early warning system, data acquired in situ must be sent automatically to a "control center" where they are integrated into a complete early warning system procedure (Intrieri et al., 2013). In this sense, another main limitation is represented by the necessity to transfer a high quantity of data, whose weight has to be reduced to the minimum, in order to reduce the load on transmission network.

The employed system is a portable device designed and implemented by the Joint Research Center (JRC) of the European Commission and its spin-off company Ellegi-LiSALab (Tarchi et al., 2003; Antonello et al., 2004).

\subsection{Early warning system architecture}

Morphological features, hydrogeological factors and sudden rainfall can cause diverse types of movements or fall of earthy and rock materials. The unpredictability and diversity of these events make structural interventions often inappropriate to reduce the related risk and a real-time monitoring network difficult to implement.

In the last decade, wireless sensor networks (WSNs) have been used extensively in various fields. A significant increase in the use of WSNs - due to their simplicity and the low cost of installation, manufacturing and maintenance - has been recorded in the framework of environmental monitoring applications (Intrieri et al., 2012; Liu et al., 2007; Yoo et al., 2007). Distinct types of sensor nodes of these networks, distributed with high density in the monitored areas, send environmental information to the concentrators nodes, generating a considerable amount and a wide variety of collected data. Due to the significant growth of data volumes to be transferred, the WSNs require flexible ad hoc protocols able to respect constraints related to energy consumption management (Hadadian and Kavian, 2016; Khaday et al., 


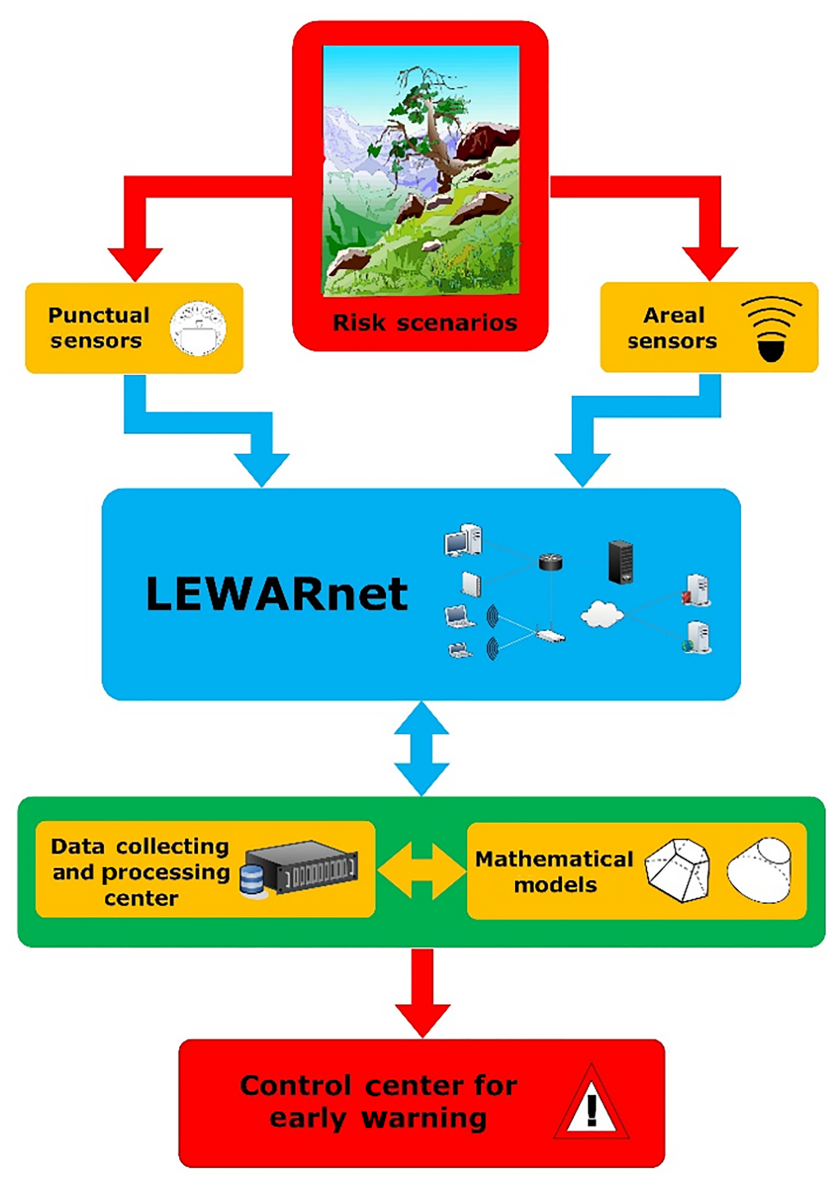

Figure 1. LEWIS architecture.

2015; Parthasarathy et al., 2015). In particular, many protocols have been developed that offer data aggregation patterns to optimize the sensor nodes' battery life (Kim et al., 2015) or sleep-measurement-data transfer cycles to minimize the energy consumption (Fei et al., 2013; Venkateswaran and Kennedy, 2013).

LEWIS (Costanzo et al., 2016) uses heterogeneous sensors, distributed in the risk areas, to monitor the several physical quantities related to landslides. The measured data, through a telecommunications network, flow into the data collecting and processing center (DCPC), where, using suitable mathematical models for the monitored site, the risk is evaluated and eventually the state of alert for mitigation action is released (Fig. 1).

The system, through a modular architecture exploiting a telecommunication network (called LEWARnet) based on an ad hoc communication protocol and an adaptive middleware, has a high flexibility, which allows for the use of different interchangeable technological solutions to monitor the parameters of interest.

The network has been equipped with both single-point sensors and area sensors. The present paper addresses a subnetwork comprising an area sensor, the GB-InSAR.
The different sensors types generate asynchronous traffic, thus imposing the adoption of an ad hoc transmission protocol. This can support an asynchronous transmission mode to the DCPC, and it is equipped with message queue management capacity to reconstruct historical data series, between two connection sessions, in case of null or partial transmission. This operation mode requires the presence of a software architecture that operates as a buffer, acting as an intermediary or as middleware (LEWARnet) between the data consumer (DCPC) and the data producers (sensors and subnetworks of sensors).

The developed middleware also monitors the processes of transmission and data acquisition, recognizing the activity status of the sensors and that of the DCPC, and integrating encryption and data compression functions.

A detailed description of LEWIS can be found in Costanzo et al. $(2015,2016)$.

\subsection{Data collecting and processing center}

The management of information flows, the telematic architecture and the services for data management are entrusted to the DCPC.

The DCPC has been designed and performed according to a complex hardware and software system able to ensure the reliability and continuity of the service, providing advance information of possible dangerous situations that may occur.

In the research project, the DCPC has to ensure the continuous exchange of information among monitoring networks, mathematical models and the command and control center (CCC) that is responsible for emergency management and decision making.

Data flow from the monitoring network was managed according to a communication protocol implemented by the DCPC and named AqSERV. AqSERV was designed considering the heterogeneity of devices of monitoring and transmission networks (single-point and area sensors) and the available hardware resources (microcontrollers and/or industrial computers). AqSERV was devised to link the DCPC database (named LEWISDB) to the monitoring networks, after validation of the authenticity of the node that connects to the center. Data acquisition, before the storage in the database, is validated both syntactically and according to the information content. The procedures for extraction of the information content and validation have been realized differently for single-point and area sensors: the latter require a more complex validation, as they work in a 2-D domain.

The complete management of the monitoring networks by DCPC has been realized through specific remote commands, sent to individual devices via AqSERV, to reconfigure the acquisition intervals or to activate any sensor, depending on the natural phenomena occurring in real time.

The configuration of monitoring networks - composed of devices and sensors, of communication protocol used by each network, and of rules for extraction and validation of infor- 


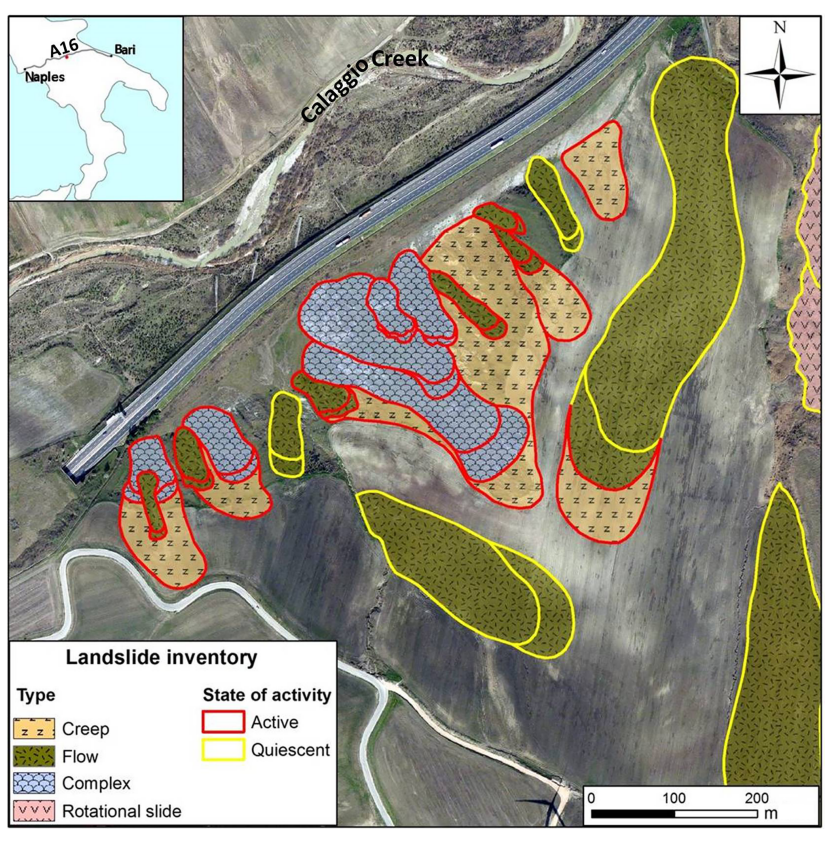

Figure 2. Landslides detected through field survey along the monitored section of A16 highway.

mation content - is carried out through a Web application that allows for the management of the entire system by the users.

The real-time search for acquisitions is carried out through a WebGIS that has been specifically designed for WSNs but that can be easily extended to classic monitoring networks.

The WebGIS was designed according to the traditional Web architecture, client-server, by using network services which are Web mapping oriented:

- Web server for static data,

- Web server for dynamic data,

- server for maps,

- database for the management of map data.

\section{Test site}

The test site chosen to experiment the integrated system is located in southern Italy, along a section of the A16 highway, an important communication route that connects Naples to Bari (Fig. 2). The selected section of the A16 highway runs in the SW-NE direction, along the southern Italian Apennines, in correspondence with the valley of the Calaggio Creek, between the towns of Lacedonia (Campania region) and Candela (Puglia region).

The area is tectonically active, but the landscape, characterized by gentle slopes, is mostly influenced by lithologic factors rather than by tectonics. The lithologies outcropping in this area are Pliocene-Quaternary clay, clayey marlstones and more recent (Holocene) terraced alluvial sediments (from clay to gravel). The landslides shown in Fig. 2 are all located in clay or clayey marlstones.

The highway runs on the right flank of the Calaggio Creek at an altitude between 300 and $400 \mathrm{~m}$ a.s.1.; the section of interest represents an element at risk in the computation of landslide risk assessment, due to the presence of unstable areas which can potentially affect the communication route (Fig. 2). These unstable areas mainly involve clayey superficial layers.

On 1 July 2014, the GB-InSAR system was installed on the test site. The location of the installation point was selected, taking into account the view of the unstable area and the distance from the power supply network. A covered structure was built to protect the system from atmospheric agents and possible acts of vandalism, in the perspective of a longterm monitoring.

The transmission network was provided by a GSM modem, exploiting the $3 \mathrm{G}$ network. In addition to the PC integrated into the GB-InSAR power base, a further external PC was exclusively employed for data after elaboration and transmission.

The system acquired data from the beginning of July 2014 until the end of July 2015.

The installation location allowed the system to detect an area between 40 and $400 \mathrm{~m}$ from the its position in the range direction and about $360 \mathrm{~m}$ wide in the azimuth direction. These values, coupled with a $40^{\circ}$ vertical aperture of the antennas, allowed operators to detect an area of about $360 \mathrm{~m} \times 360 \mathrm{~m}$.

\section{Data management}

The most relevant matter of this monitoring was not as much related to the detection of landslide movements threatening the highway as to how long-term monitoring performed with an instrument providing huge amounts of data could have been run without resorting to large hard drives or to fast Internet connections. In fact, the monitoring area was covered by a $3 \mathrm{G}$ mobile telecommunication networks with a limit of 2-gigabyte data transfer per month, and there was the need to reduce the massive data flow produced by the radar.

For this reason, an appropriate data management system (Fig. 3) was developed and is described herein.

\subsection{Data acquisition}

The GB-InSAR employed produced a single radar image, consisting of a $1001 \times 1001$ complex matrix, every $5 \mathrm{~min}$. Each one is around 8 megabytes large, resulting in more than 2 gigabytes of data produced every day.

This amount of data represented an issue for both store capacity and data transmission. 


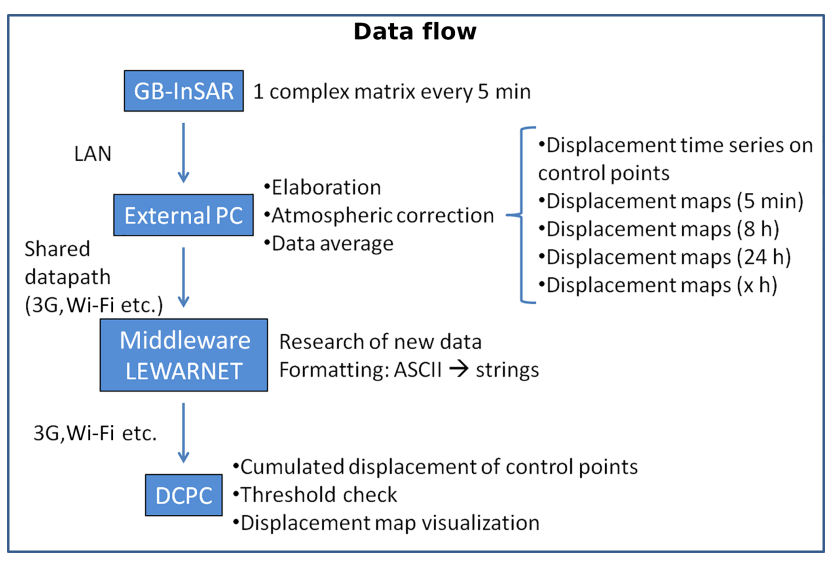

Figure 3. Diagram showing the complete data flow from acquisition to final visualization.

\subsection{Data elaboration}

After being acquired, data were then transferred through LAN connection to the external PC implementing a dedicated Matlab script locally performing the actions described as follows.

\subsubsection{Data averaging}

In order to reduce the noise normally affecting radar data (especially in vegetated areas), the images acquired every 5 min were also averaged using all data of the previous 8 and $24 \mathrm{~h}$. Then images averaged over $24 \mathrm{~h}$ were used to calculate daily displacement maps every $8 \mathrm{~h}$ to create $8 \mathrm{~h}$ displacement maps, and non-averaged images were used to calculate 5 min displacement maps. These time frames were selected based on the characteristics of the slope movements and signal/noise ratio in the investigated area.

Averaging is also a way to make good use of a high data frequency, since it enables the memory occupied in the database to be reduced as an alternative to their direct elimination.

\subsubsection{Displacement map calculation and ASCII conversion}

Each radar image can be represented as in Eq. (1):

$S_{n}=A_{n} \exp \left(j \varphi_{n}\right) m$

where $A_{n}$ is the amplitude of the $n$th image, $\varphi_{n}$ is its phase and $j=(-1)^{1 / 2}$ is the imaginary unit. The displacement $\Delta r$ occurred in the time period between the acquisition of $S_{1}$, and $S_{2}$ was calculated with the following (Eq. 2):

$\Delta r=(\lambda / 4 \pi) \cdot \Delta \varphi$,

where $\lambda$ is the wavelength of the signal and

$\Delta \varphi=\varphi_{1}-\varphi_{2}$ can be derived from

$S_{3}=S_{1} S_{2}^{*}=A_{1} A_{2} \exp \left[j\left(\varphi_{1}-\varphi_{2}\right)\right]$.

As a result, an ASCII file was obtained that only contains the information relative to the displacement for each pixel.

\subsubsection{Atmospheric correction}

One of the major advantages of GB-InSAR is the capability to achieve sub-millimeter precision. However, this can be severely hampered by the variations of air temperature and humidity, especially when long distances are involved. Usually, atmospheric correction is performed by choosing one area considered stable, taking into account that every displacement value different from 0 is due to atmospheric noise and assuming that this offset is a linear function of the distance. Based on this relation the entire displacement map is corrected. In our case the whole scenario has been selected, and then only the potentially unstable zones and those with a weak or incoherent backscattered signal were removed. The remaining areas were then considered stable and therefore were used for calculating the atmospheric effects. This results in a larger correction region that enables a statistical correlation between the atmospheric effects and the distance and therefore the calculation of a site-specific regression function that may not necessarily be linear (Fig. 4).

\subsubsection{Line-of-sight correction}

The availability to detect only the line-of-sight (LOS) component of the displacement vector represents one of the main limitations of the GB-InSAR technique. A method to partially overcome this limitation has been applied in this paper, following the procedure described in Colesanti and Wasowski (2006) and later in Bardi et al. (2014, 2016). Other methods have been employed by Cascini et al. (2010, 2013).

Assuming the downslope direction as the most probable displacement path, radar data have been projected in this direction. Input data as the angular values of the aspect and slope have been derived from the digital terrain model (DTM) of the investigated area; furthermore, azimuth angle and incidence angle of the radar LOS have been obtained.

After calculating the direction cosines of LOS and slope (respectively functions of azimuth and incidence angles and aspect and slope angles) in the directions of zenith $\left(Z_{\mathrm{los}}\right.$, $\left.Z_{\text {slope }}\right)$, north $\left(N_{\text {los }}, N_{\text {slope }}\right)$ and east $\left(E_{\text {los }}, E_{\text {slope }}\right)$, the coefficient $C$ is defined as follows (Eq. 5):

$C=Z_{\text {los }} \times Z_{\text {slope }}+N_{\text {los }} \times N_{\text {slope }}+E_{\text {los }} \times E_{\text {slope }}$.

$C$ represents the percentage of real displacement detected by the radar sensor (Fig. 5a).

The real displacement $\left(D_{\text {real }}\right)$ is defined as the ratio between the displacement recorded along the LOS $\left(D_{\text {los }}\right)$ and the $C$ value (Fig. 5b). 
(a)

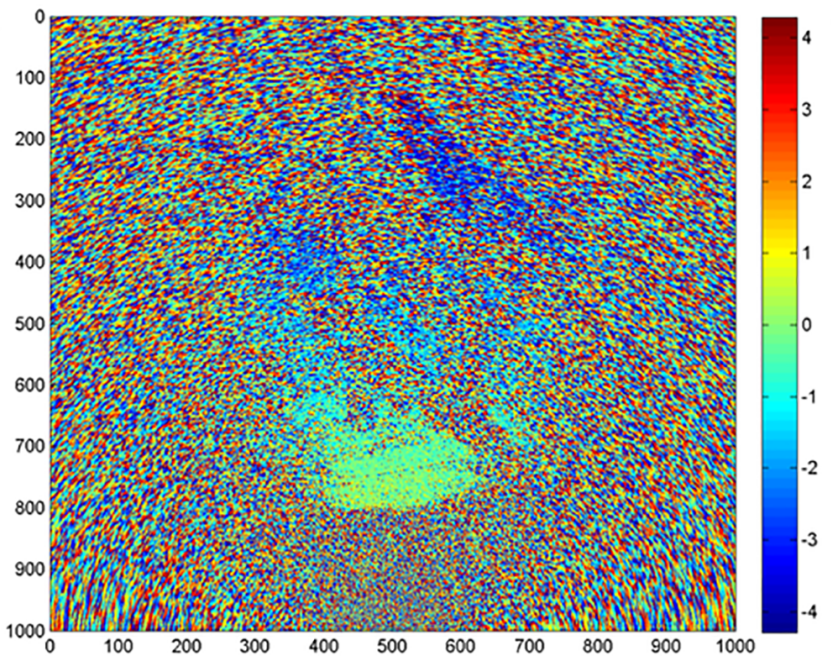

(b)

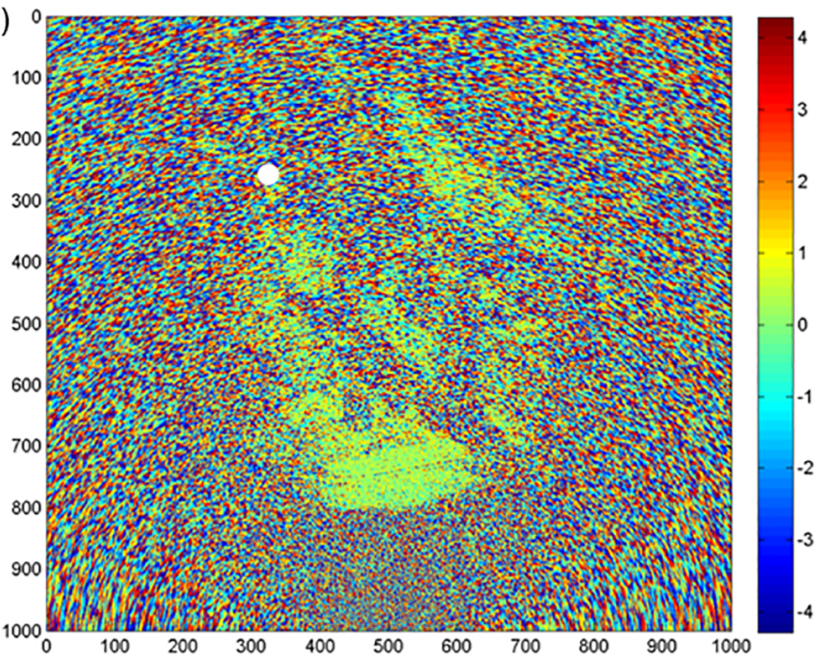

Figure 4. The color bar is expressed in millimeters; green indicates stable pixels, while blue and red respectively indicate movement toward and away from the GB-InSAR. (a) Raw interferogram showing artificial displacement increasing linearly with distance (as typical of atmospheric noise). (b) The same interferogram after the atmospheric correction.

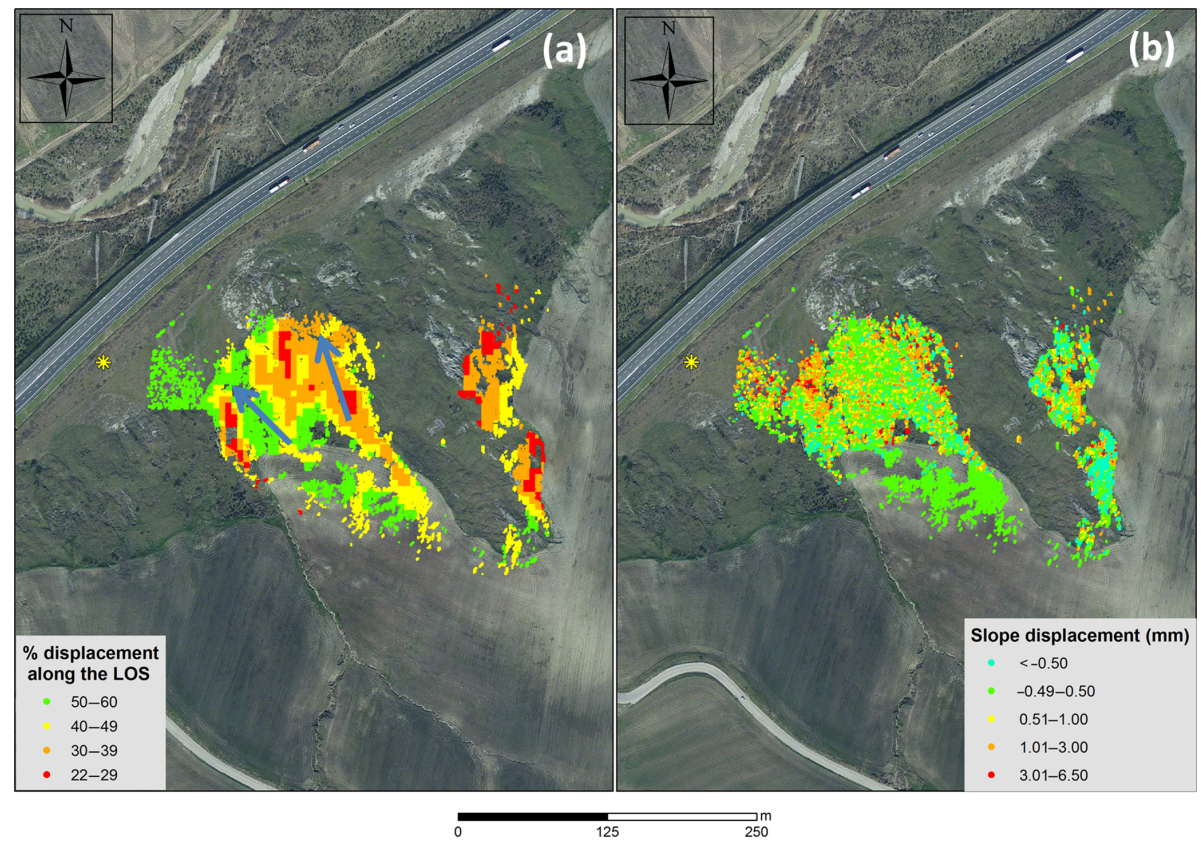

Figure 5. (a) $C$ values map. Blue arrows indicate the downslope direction. (b) Cumulated displacement values projected in the downslope direction, referring to a period between 1 July and 1 November 2014. The yellow asterisk on the left of the images represents the location of the GB-InSAR.

Assuming that the studied landslide actually moves in the downslope direction, the GB-InSAR detectable real displacement percentage ranges between 22 and $60 \%$ (Fig. 5a).

In Fig. 5b, an example of a slope displacement map is shown. Here, cumulated displacement data related to a period between 1 July and 1 November 2014 have been projected in the downslope direction. Data show that the area can be considered stable in the referred period; maximum displacement values of $4 \mathrm{~mm}$ in 4 months (eastern portion of observed scenario) can be still considered in the range of stability.

\subsubsection{Time series extraction}

In order to allow for a fast data transfer-velocity threshold comparison, some representative control points were selected, aimed at providing cumulated displacement time series. Control points were retrieved from the same displace- 
ment maps calculated as described in Sect. 5.2.2 and therefore can be relative to a time frame of $5 \mathrm{~min}, 8 \mathrm{~h}$ or $24 \mathrm{~h}$.

In the case of noisy data, instead of having a time series relative to a single pixel, these can be retrieved from a spatial average obtained from a small area consisting of few pixels.

\subsubsection{Scenario cropping}

Typically, the field of view of a GB-InSAR is larger than the actual area to be monitored. In fact, a portion of the radar image may be relative to the ground, sky and areas geometrically shadowed or covered by dense vegetation. These may be of no interest or even contain no information at all. For the case here studied around $50 \%$ of a radar image had a low coherence and was for all practical purposes unusable. Therefore, a cropping of the ASCII displacement map occurred in order to frame only the relevant area.

\subsection{Data transfer and visualization}

The interferometric data generated by GB-InSAR, after the pre-processing and proper correction previously described, are ready for transfer to the DCPC. The transmission of these data to the DCPC is mediated by the middleware, which interrogates the GB-InSAR in order to track the state, detects the newest data, and reorders and marks them to properly build data time series to be transferred to DCPC.

Subsequently, the middleware manages communications with the DCPC, according to the implemented ad hoc protocol. This ensures the security of data providers through encrypted authentication mechanisms; it allows for recovering missing or partially transmitted data, thus avoiding information loss; and it provides data acquired by the sensors to the DCPC in a standardized format, JSON, able to guarantee uniformity between the various information provided by the various sensors types. All these particular features fully justify the adoption of an ad hoc protocol for data transfer, instead of using a standard protocol such as FTP.

The data files produced by the GB-InSAR have already been locally pre-processed and result in a matrix expressed in ASCII code; the dimensions of the matrix are known and range from $1 \times 1$ (for the displacement of single control points) to $1001 \times 1001$ (for uncropped displacement maps). Before encapsulating these data in the message to be transferred to DCPC, the middleware converts them from ASCII code to character strings, using the standard coding ISO/IEC 8859-1, and so is able to obtain a data compression with a factor equal to $\approx 8$.

Eventually the DCPC is entrusted with cumulating the displacements relative to the control points, which are compared with the respective thresholds, and with visualizing the displacement maps as WebGIS layers, thus enabling data validation and the evaluation of the extension of moving surface.

\section{Early warning procedure discussions}

The GB-InSAR is part of a larger early warning system (LEWIS) which also includes other monitoring systems and simulation models. Therefore, to understand how GB-InSAR data can be used in an early warning context, it is necessary to make reference to LEWIS as a whole.

Any information coming from the investigated sites and subsequently processed also by using the simulation models is used to define an intervention model. This is based on the following elements: event scenarios, risk scenarios, levels of criticality and levels of alert.

Event scenarios describe the properties of expected phenomena in terms of dimension, velocity, involved material and occurrence probability. Occurrence probability depends on the associated time horizon, which should be equal to a few hours at most, in the case of early warning systems. Evaluation of occurrence probability is carried out by using information from monitoring systems and/or from outputs of adopted mathematical models for nowcasting. All the properties to be analyzed for event scenarios are listed below; a subdivision in classes is adopted for each one:

- landslide velocity (five classes from slow to extremely rapid);

- landslide surface (five classes from very small to very large);

- landslide scarp (five classes from very small to very large);

- landslide volume (five classes from extremely small to large);

- thickness (five classes from very shallow to very deep);

- magnitude (three classes: low, moderate, high), which combines the previous information;

- involved material (mud, debris, earth, rock, mixture of components);

- occurrence probability (zero, low, moderate, high, very high, equal to 1 ).

While some of the aforementioned parameters are determined by geological surveys, landslide velocity is directly derived from monitoring data (such as those collected by GB-InSAR). Landslide surface can be determined by geomorphological observation but is precisely quantified by GBInSAR, thanks to its capability to produce 2-D displacement maps.

Risk scenarios can be firstly grouped in the following three classes:

A. mud and/or debris movements which could induce a friction reduction between the vehicles and the tar and therefore facilitate slips; 


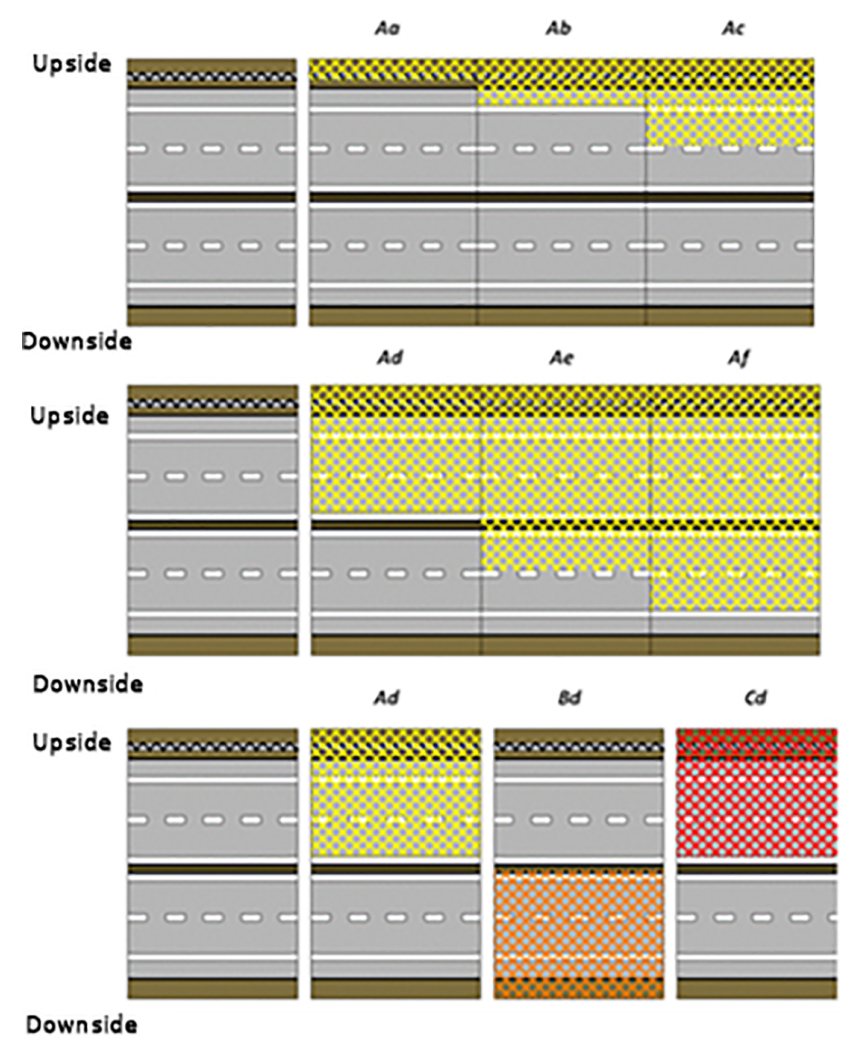

Figure 6. Top and middle: possible risk scenarios involving scenario A (landslides that could reduce friction) to increasing sectors of the highway. Bottom: combinations of scenarios with several types of phenomena that affect the emergency lane, regular lane and fast lane.

B. road subsidence induced by landslides that could drag or drop vehicles;

C. falls of significant volumes and/or boulders that could crush or cover vehicles and constitute an obstacle for other vehicles.

For each previous risk scenario, six sub-scenarios can be identified based on the number of potentially involved infrastructures, carriageways and lanes (a: hydraulic infrastructures and/or barriers of either carriageway; b: only the emergency lane of either carriageway; c: the emergency lane and up to the regular lane of either carriageway; d: up to the fast lane of either carriageway; e: up to the fast lane of the opposite carriageway; f: up to the regular lane of the opposite carriageway). Thus, the total number of possible risk scenarios is 18 (Fig. 6), indicated with a couple of letters (capital and small).

The following information is provided to DCPC:

- measurements from sensors,

- model outputs,

and four states are identified for each of them:
- state 0: no variation,

- state 1: small variation,

- state 2: moderate variation,

- state 3: high variation.

In practice, for the GB-InSAR, such states are delimited by fixed velocity values (thresholds). In this application values have been selected according to the gathered data, the first threshold being just above the instrumental noise; the remaining have been set based on expert judgement waiting for a more robust calibration, which is possible only after at least a partial mobilization of the slope. Anyway, the system is open to any method for determining thresholds (Crosta and Agliardi, 2003; Du et al., 2013; Carlà et al., 2016a) and also to the use of other parameters (acceleration for example).

Besides information from sensors and models, other information is obtained from meteorological and hydrological models (named "indicators").

Indicators comprise weather forecasting and output of the Forecasting of Landslides Induced by Rainfall (FLaIR) and Saturated Unsaturated Simulation for Hillslope Instability (SUSHI) models (Sirangelo et al., 2003; Capparelli and Versace, 2011) on the basis of observed and predicted (for the successive $6 \mathrm{~h}$ ) rainfall heights.

Two states are defined for indicators:

- state 0: no variation or not significant,

- state 1: significant variation.

To sum up, DCPC has the following information at any moment:

- state $(0,1)$ of indicators (INDs),

- state $(0,1,2,3)$ of sensors and models running for the specific highway section (SEN),

and, on the basis of these states, four different decisions can be made by DCPC, one of which with three options.

All the possible decisions are illustrated in Table 1, in which the weight of the several sensors is assumed to be the same. Based on the notices of criticality levels provided by the DCPC, and on its own independent evaluations, the CCC issues the appropriate warning notices (surveillance, alert, alarm and warning) and makes decisions about the consequent actions.

The information of each sensor and the results produced by the models are used to assess, in each instant, the occurrence probability of an event scenario in the monitored areas and the possible risk scenarios.

This combination of heterogeneous data was carried out by identifying for each sensor and model typical information (displacement, precipitation, inclination etc.); evaluating the state at each instant, according to a threshold system; and 
Table 1. DCPC possible decisions.

\begin{tabular}{ll}
\hline State of sensors and/or models & DCPC decisions \\
\hline All INDs and SENs are S0 & $0-$ no decision \\
At least one IND is S1 and all SENs are S0 & $1-$ sensor on demand activation \\
At least one SEN is S1 & $2-$ to intensify the presence up to 24h a day \\
At least $n$ SENs are S1 or at least one SEN is S2 & $3 / 1-$ to issue a notice of ordinary criticality (level 1) \\
At least $n$ SENs are S2 or at least one SEN is S3 & $3 / 2-$ to issue a notice of moderate criticality (level 2) \\
At least $n$ SENs are S3 & $3 / 3$ - to issue a notice of high or severe criticality (level 3) \\
\hline
\end{tabular}

combining this result for all sensors placed in a monitored geomorphological area.

The result is constituted by the occurrence probability of an event scenario, which is associated with a specific action by the DCPC. In particular, if the occurrence probability is low, moderate or high, it is necessary to issue a notice of criticality (ordinary - level 1; moderate - level 2; high - level 3) to the CCC.

The DCPC sends two types of information:

1. criticality state of the single monitored geomorphological unit,

2. criticality state of the whole area.

The adopted communication protocol between the two centers for the exchange of information was carried out through a Web service provided by the CCC, using the classes and attributes of the methodology named DATEX II (which is a protocol for the exchange of traffic data). The use of the Web service allowed ensuring the interoperability of data between the two centers, regardless of the hardware and software architecture used, through a persistent service capable of ensuring an immediate restoration of the connections, in case of malfunction, and a continuous monitoring between the two centers, even in the absence of criticality.

\section{Conclusions}

The GB-InSAR is a monitoring tool that is becoming more and more used in landslide monitoring and early warning, especially thanks to its capability to produce real-time, 2-D displacement maps. On the other hand, it still suffers from some drawbacks, such as the limitation of measuring only the LOS component of a target's movement and logistic issues like those owing to a massive production of data that may cause trouble for both storing capacity and data transfer. In particular, the latter is a more and more common problem of advanced technologies that are able to produce high-quality data with a high acquisition frequency, which may leave the problem of finding the balancing between exploiting all the information and at the same time avoiding unnecessary redundancy.

These problems were addressed when a GB-InSAR was integrated within a complex early warning system (LEWIS) and only a limited Internet connection was available. This situation required that a series of pre-elaboration processes and data management procedures take place in situ in order to produce standardized and reduced files, carrying only the information needed when it was needed. The procedures mainly concerned the transmission of data averaged over determined time frames, proportionate with the kinematics of the monitored phenomenon. Previously, transmission data were also corrected (in terms of both atmospheric noise and LOS) and reduced, by filtering out the information relative to the amplitude of the targets, by eliminating the areas not relevant for the monitoring and by transforming the matrices into strings.

As a result, GB-InSAR data converged into the early warning system and contributed to it by producing displacement time series of representative control points to be compared with fixed thresholds. Displacement maps were also available for data validation by expert operators and for retrieving information relative to the surface of the moving areas.

Data availability. The present paper concerns a methodology. Some of the data used in this paper are no longer available; the remaining available data can be requested from the corresponding author.

Competing interests. The authors declare that they have no conflict of interest.

Special issue statement. This article is part of the special issue "Landslide early warning systems: monitoring systems, rainfall thresholds, warning models, performance evaluation and risk perception". It is not associated with a conference.

Acknowledgements. This research is part of the project "LEWIS (Landslides Early Warning Integrated System): An Integrated System for Landslide Monitoring, Early Warning and Risk Mitigation along Lifelines", financed by the Italian Ministry of Education, Universities and Research and co-funded by the European Regional Development Fund, in the framework of the National Operational Programme 2007-13 "Research and Competitiveness", grant agreement no. PON01_01503. 
The authors are thankful to Giuseppe Della Porta and his colleagues from Autostrade S.p.A. for their availability in permitting and supporting the installation and maintenance of the GB-InSAR along the A16 highway.

Edited by: Stefano Luigi Gariano

Reviewed by: two anonymous referees

\section{References}

Antonello, G., Casagli, N., Farina, P., Leva, D., Nico, G., Sieber, A. J., and Tarchi, D.: Ground-based SAR interferometry for monitoring mass movements, Landslides, 1, 21-28, 2004.

Baldridge, S. M. and Marshall, J. D.: Performance of structures in the January 2010 MW 7.0 Haiti earthquake, Structures Congress, 1660-1671, https://doi.org/10.1061/41171(401)145, 2011.

Bamler, R. and Hartl, P.: Synthetic Aperture Radar Interferometry, Inverse Probl., 14, R1-R54, 1998.

Bardi, F., Frodella, W., Ciampalini, A., Del Ventisette, C., Gigli, G., Fanti, R., Basile, G., Moretti, S., and Casagli, N.: Integration between ground based and satellite SAR data in landslide mapping: The San Fratello case study, Geomorphology, 223, 45-60, 2014.

Bardi, F., Raspini, F., Ciampalini, A., Kristensen, L., Rouyet, L., Lauknes, T. R., Frauenfelder, R., and Casagli, N.: Space-Borne and Ground-Based InSAR Data Integration: The Åknes Test Site, Remote Sens.-Basel., 8, 237, https://doi.org/10.3390/rs8030237, 2016.

Bardi, F., Raspini, F., Frodella, W., Lombardi, L., Nocentini, M., Gigli, G., Morelli, S., Corsini, A., and Casagli, N.: Monitoring the Rapid-Moving reactivation of Earth Flows by Means of GB-InSAR: The April 2013 Capriglio Landslide (Northern Appennines, Italy), Remote Sens.-Basel., 9, 165, https://doi.org/10.3390/rs9020165, 2017.

Cagno, E., De Ambroggi, M., Grande, O., and Trucco, T.: Risk analysis of underground infrastructures in urban areas, Reliab. Eng. Syst. Safe, 96, 139-148, 2011.

Calvari, S., Intrieri, E., Di Traglia, F., Bonaccorso, A., Casagli, N., and Cristaldi, A.: Monitoring crater-wall collapse at active volcanoes: a study of the 12 January 2013 event at Stromboli, B. Volcanol., 78, 1-16, https://doi.org/10.1007/s00445-016-1033-4, 2016.

Capparelli, G. and Versace, P.: FLaIR and SUSHI: Two mathematical models for early warning of landslides induced by rainfall, Landslides, 8, 67-79, 2011.

Carlà, T., Intrieri, E., Di Traglia, F. and Casagli, N.: A statisticalbased approach for determining the intensity of unrest phases at Stromboli volcano (Southern Italy) using one-step-ahead forecasts of displacement time series, Nat. Hazards, 84, 669-683, $2016 a$.

Carlà, T., Intrieri, E., Di Traglia, F., Nolesini, T., Gigli, G., and Casagli, N.: Guidelines on the use of inverse velocity method as a tool for setting alarm thresholds and forecasting landslides and structure collapses, Landslides, 14, 517-534, $2016 \mathrm{~b}$.

Cascini, L., Fornaro, G., and Peduto, D.: Advanced low- and full-resolution DInSAR map generation for slowmoving landslide analysis at different scales, Eng. Geol., 112, 29-42, https://doi.org/10.1016/j.enggeo.2010.01.003, 2010.
Cascini, L., Peduto, D., Pisciotta, G., Arena, L., Ferlisi, S., and Fornaro, G.: The combination of DInSAR and facility damage data for the updating of slow-moving landslide inventory maps at medium scale, Nat. Hazards Earth Syst. Sci., 13, 1527-1549, https://doi.org/10.5194/nhess-13-1527-2013, 2013.

Colesanti, C. and Wasowski, J.: Investigating landslides with spaceborne Synthetic Aperture Radar (SAR) interferometry, Eng. Geol., 88, 173-199, 2006.

Costanzo, S., Di Massa, G., Costanzo, A., Morrone, L., Raffo, A., Spadafora, F., Borgia, A., Formetta, G., Capparelli, G., and Versace, P.: Low-cost radars integrated into a landslide early warning system, Adv. Intell. Syst., 354, 11-19, 2015.

Costanzo, S., Di Massa, G., Costanzo, A., Borgia, A., Raffo, A., Viggiani, G. and Versace, P.: Software-defined radar system for landslides monitoring, Adv. Intell. Syst., 445, 325-331, 2016.

Crosta, G. B. and Agliardi, F.: How to obtain alert velocity thresholds for large rockslides, Phys. Chem. Earth., Pt. A/B/C, 27, 1557-1565, 2002.

Del Ventisette, C., Intrieri, E., Luzi, G., Casagli, N., Fanti, R., and Leva, D.: Using ground based radar interferometry during emergency: the case of the A3 motorway (Calabria Region, Italy) threatened by a landslide, Nat. Hazards Earth Syst. Sci., 11, 2483-2495, https://doi.org/10.5194/nhess-11-2483-2011, 2011.

Di Traglia, F., Nolesini, T., Intrieri, E., Mugnai, F., Leva, D., Rosi, M., and Casagli N.: Review of ten years of volcano deformations recorded by the ground-based InSAR monitoring system at Stromboli volcano: a tool to mitigate volcano flank dynamics and intense volcanic activity, Earth-Sci. Rev., 139, 317-335, 2014.

Du, J., Yin, K., and Lacasse, S.: Displacement prediction in colluvial landslides, three Gorges reservoir, China, Landslides, 10, 203-218, 2013.

Fei, X., Zheng, Q., Tang, T., Wang, Y., Wang, P., Liu, W., and Yang, H.: A reliable transfer protocol for multi-parameter data collecting in wireless sensor networks, 2013 15th Int Conf Adv Commun: Smart Services with Internet of Things, ICACT 2013, 569573, 2013.

Geertsema, M., Schwab, J. W., Blais-Stevens, A., and Sakals, M. E.: Landslides impacting linear infrastructure in west central British Columbia, Nat. Hazards, 48, 59-72, 2009.

Gene Corley, W., Mlakar, P. F. Sr., Sozen, M. A., and Thornton, C. H.: The Oklahoma City bombing: Summary and recommendations for multihazard mitigation, J. Perform. Constr. Fac., 12, 100-112, 1998.

Hadadian, H. and Kavian, Y.: Cross-layer protocol using contention mechanism for supporting big data in wireless sensor network, 2016 10th International Symposium on Communication Systems, Networks and Digital Signal Processing (CSNDSP), 2016.

Intrieri, E., Gigli, G., Mugnai, F., Fanti, R., and Casagli, N.: Design and implementation of a landslide early warning system, Eng. Geol., 147-148, 124-136, 2012.

Intrieri, E., Gigli, G., Casagli, N., and Nadim, F.: Brief communication "Landslide Early Warning System: toolbox and general concepts", Nat. Hazards Earth Syst. Sci., 13, 85-90, https://doi.org/10.5194/nhess-13-85-2013, 2013.

Intrieri, E., Gigli, G., Nocentini, M., Lombardi, L., Mugnai, F., and Casagli, N.: Sinkhole monitoring and early warning: An experimental and successful GB-InSAR application, Geomorphology, 241, 304-314, 2015. 
Kadri, F., Birregah, B., and Châtelet, E.: The impact of natural disasters on critical infrastructures: A domino effect-based study, J. Homel. Secur. Emerg., 11, 217-241, 2014.

Khaday, B., Matson, E. T., Springer, J., Kwon, Y. K., Kim, H., Kim, S., Kenzhebalin, D., Sukyeong, C., Yoon, J., and Woo, H. S.: Wireless Sensor Network and Big Data in Cooperative Fire Security system using HARMS, 2015 6th International Conference on Automation, Robotics and Applications (ICARA), 2015.

Kim, Y., Bae, P., Han, J., and Ko, Y. B.: Data aggregation in precision agriculture for low-power and lossy networks, 2015 IEEE Pacif, 2015.

Kröger, W.: Critical infrastructures at risk: A need for a new conceptual approach and extended analytical tool, Reliab. Eng. Syst. Safe, 93, 1781-1787, 2008.

Labaka, L., Hernantes, J., and Sarriegi, J. M.: A holistic framework for building critical infrastructure resilience, Technol. Forecast Soc., 103, 21-33, 2016.

Liu, H., Meng, Z., and Cui S.: A Wireless Sensor Network Prototype for Environmental Monitoring in Greenhouses, 2007 Int C Wirel Comm Net, 2007.

Lombardi, L., Nocentini, M., Frodella, W., Nolesini, T., Bardi, F., Intrieri, E., Carlà, T., Solari, L., Dotta, G., Ferrigno, F., and Casagli, N.: The Calatabiano landslide (southern Italy): preliminary GB-InSAR monitoring data and remote 3D mapping, Landslides, 14, 1-12, 2016.

Luzi, G.: Ground Based SAR Interferometry: a novel tool for geoscience, in: Geoscience and Remote Sensing. New Achievements, edited by: Imperatore, P. and Riccio, D., InTech, available at: http://www.intechopen.com/articles/show/title/ ground-based-sar-interferometry-a-novel-tool-for-geoscience, 1-26, https://doi.org/10.5772/9090, 2010.

Martino, S. and Mazzanti, P.: Integrating geomechanical surveys and remote sensing for sea cliff slope stability analysis: the Mt. Pucci case study (Italy), Nat. Hazards Earth Syst. Sci., 14, 831848, https://doi.org/10.5194/nhess-14-831-2014, 2014.

Monserrat, O., Crosetto, M., and Luzi, G.: A review of groundbased SAR interferometry for deformation measurement, ISPRS J. Photogramm, 93, 40-48, 2014.

Parthasarathy, A., Chaturvedi, A., Kokane, S., Warty, C., and Nema, S.: Transmission of big data over MANETs, Aerosp Conf Proc, 2015.

Rudolf, H., Leva, D., Tarchi, D., and Sieber, A. J.: A mobile and versatile SAR system, IGARSS Proc., Hamburh, 1999.

Severin, J., Eberhardt, E., Leoni, L., and Fortin, S.: Development and application of a pseudo-3D pit slope displacement map derived from ground-based radar, Eng. Geol., 181, 202-211, 2014.
Sirangelo, B., Versace, P., and Capparelli, G.: Forewarning model for landslides triggered by rainfall based on the analysis of historical data file, IAHS-AISH P., 278, 298-304, 2003.

Snyder, L. and Burns, A. A.: Framework for critical infrastructure resilience analysis. Energy and systems analysis-infrastructure, Sandia National Laboratories, 2009.

Tapete, D., Casagli, N., Luzi, G., Fanti, R., Gigli, G., and Leva, D.: Integrating radar and laser-based remote sensing techniques for monitoring structural deformation of archaeological monuments, J. Archaeol. Sci., 40, 176-189, 2013.

Tarchi, D., Ohlmer, E., and Sieber, A. J.: Monitoring of structural changes by radar interferometry, Res. Nondestruct. Eval., 9, 213 225, 1997.

Tarchi, D., Rudolf, H., Luzi, G., Chiarantini, L., Coppo, P., and Sieber, A. J.: SAR interferometry for structural change detection: a demonstration test on a dam, Int. Geosci. Remote Sens., 3, 1525-1527, 1999.

Tarchi, D., Casagli, N., Fanti, R., Leva, D., Luzi, G., Pasuto, A., Pieraccini, M., and Silvano, S.: Landslide monitoring by using ground-based SAR interferometry: an example of application to the Tessina landslide in Italy, Eng. Geol., 68, 15-30, 2003.

Urlainis, A., Shohet, I. M., Levy, R., Ornai, D., and Vilnay, O.: Damage in critical infrastructures due to natural and man-made extreme Events - A critical review, Procedia Engineer, 85, 529535, 2014.

Urlainis, A., Shohet, I. M., and Levy, R.: Probabilistic Risk Assessment of Oil and Gas Infrastructures for Seismic Extreme Events, Procedia Engineer, 123, 590-598, 2015.

Venkateswaran, V. and Kennedy, I.: How to sleep, control and transfer data in an energy constrained wireless sensor network, 51st Annual Allerton Conference on Communication, Control, and Computing (Allerton), 2013.

Versace, P., Capparelli, G., Leone, S., Artese, G., Costanzo, S., Corsonello, P., Di Massa, G., Mendicino, G., Maletta, D., Muto, F., Senatore, A., Troncone, A., Conte, E., and Galletta, D.: LEWIS project: An integrated system of monitoring, early warning and mitigation of landslides risk, Rendiconti Online Società Geologica Italiana, 21, 586-587, 2012.

Yoo, S., Kim, J., Kim, T., Ahn, S., Sung, J. and Kim, D.: A2S: Automated Agriculture System based on WSN, I Symp. Consum Electr., 2007. 